\section{O Fator Acidentário Previdenciário como instrumento epidemiológico de controle de riscos do trabalho*}

\section{The work accident and social security factor as an epidemiologic tool for controlling risks in work settings}

\section{Heleno Rodrigues Corrêa Filho \\ Departamento de Medicina Preventiva e Social, Faculdade de Ciências Médicas, Universidade Estadual de Campinas (UNICAMP)}

*Versão preliminar publicada na Revista Conjuntura Social do Ministério da Previdência Social, 2004;(03)

Correspondência: Rua Tessália Vieira de Camargo, 126, Cidade Universitária Zeferino Vaz. CEP13084-970 Campinas -SP. E-mail: helenocf@unicamp.br

Trabalho financiado pelo Ministério da Previdência Social - Secretaria de Previdência Social, agosto de 2004

\section{Resumo}

Este trabalho discute a validade da Resolução Ministerial $n^{\circ} 1.236$, do Conselho Nacional de Previdência Social, que dispõe sobre padrões de freqüência, gravidade e custo no cálculo do Fator Acidentário Previdenciário. Esse fator multiplicará alíquotas de impostos sobre riscos decorrentes do trabalho, podendo reduzir em até $50 \%$ o imposto devido por empresas que aplicarem controles coletivos eficientes na sua prevenção. Analisa-se a consistência do método adotado para selecionar os grupos de morbidade destinados a construir os vetores de freqüência, gravidade e custo, bem como a validade da aplicação dos conceitos epidemiológicos à produção econômica geradora da morbidade. Contrapõem-se as listas tradicionais de morbidade aos critérios epidemiológicos móveis, como alternativas distintas que têm sido adotadas para resolução de conflitos sobre a existência de nexo causal entre doenças, lesões, acidentes e os modos de produção. Apoiam-se os grupos de riscos epidemiológicos móveis que correspondam às doenças e lesões cujo risco epidemiológico, medido por um intervalo de confiança de $99 \%$, esteja acima do valor unitário. Concluise que o método proposto para determinação do Fator multiplicador atende aos requisitos epidemiológicos de definição de cálculo de riscos e validade, uma vez que é assegurada sua revisão periódica para questões de sensibilidade e especificidade. Alerta-se que não se deve confiar unicamente nos métodos como instrumentos unilaterais de ação social para avaliação, controle e prevenção dos males associados ao trabalho nas modalidades de produção do nosso desenvolvimento. Os métodos são instrumentos que devem ser considerados na tomada de decisão e na ação política que se deseja imprimir.

Palavras-chave: Saúde de Trabalhadores. Previdência Social. Impostos. Medição de Risco. Estudos de Coortes. Bases de Dados/ Utilização. Registro Médico Coordenado. 


\section{Abstract}

This report discusses the validity of a Brazilian Ministry Decree that establishes standards for calculating a multiplying Factor for Accidents and Social Security. The index estimates the frequency, severity and costs to be accounted for in tax calculation. It multiplies tax rates due to work risks and may reduce by up to $50 \%$ the taxes due by companies that apply efficient collective methods for disease prevention. The method is analyzed for its consistency in selecting groups of morbidity used as a mean to build frequency, severity and cost burden vectors. The validity of the application of epidemiologic concepts to the economic activity that generates the specific morbidity is also assessed. The discussion addresses the antagonism between the concepts of traditional morbidity lists and the mobile epidemiologic criteria usually adopted to solve conflicts on the existence of a causal link amongst diseases, lesions, accidents and means of production. The floating groups of morbidity that reach significant $99 \%$ confidence intervals for epidemiologic risks above the null value_1.0_are supported. The conclusion is that the method is in accordance with epidemiologic requirements, as long as a systematic review panel assesses aspects of sensitivity and specificity. A warning is presented not to rely solely on methods as unilateral means of social action for evaluating, controlling and preventing the diseases associated with the means of production that are inherent to our social development. Methods are instruments that ought to be considered in decision-making and in the desired political action.

Keywords: Workers' Health. Social Security. Taxes. Risk assessment. Cohort studies. Databases/utilization. Medical Record Linkage.

\section{Introdução}

A Resolução Ministerial no 1.236, de 28 de abril de 2004, do Conselho Nacional de Previdência Social, sancionada pelo Presidente do Conselho, o Ministro da Previdência Social em 10 de maio de $2004^{1}$, dispõe sobre padrões de freqüência, gravidade e custo no estabelecimento de Fator Acidentário Previdenciário (FAP) que multiplicará as alíquotas de impostos sobre riscos decorrentes do trabalho (1, 2 e $3 \%)$.

O FAP poderá reduzir em até $50 \%$ o imposto devido por empresas que aplicarem controles coletivos eficientes que permitam prevenir e reduzir doenças em seus trabalhadores. Poderá também aumentar em até 2 vezes as alíquotas incidentes em caso de não atuar sobre os ambientes de trabalho e sobre o modo de produção, de tal maneira que os seus indicadores de morbidade previdenciária sejam piores que os de seu próprio ramo de atividade econômica ou mais freqüentes, graves e custosos que os demais setores produtivos de outros códigos de atividade econômica.

As bases técnicas do FAP para a construção de escala contínua de 0,5 a 2. São estruturadas a partir da seleção de diagnósticos médicos de benefícios por doença ou por acidente, independentemente do reconhecimento e da notificação de nexo causal com o trabalho.

As doenças e lesões selecionadas passam a compor um agrupamento de morbidade específico para a categoria da Classificação de Atividade Econômica. Em função desse grupo são calculados coeficientes de freqüência de benefícios iniciados, gravidade ou duração total dos benefícios iniciados e custo proporcional à arrecadação potencial da Previdência Social, que ocorreria caso não necessitasse pagar o benefício.

Os três coeficientes são combinados em análise tridimensional como vetores espaciais cuja faixa de variabilidade é estimada. As empresas são então agrupadas em 3 conglomerados ou centróides, para os valores de 1 , 2 e $3 \%$, segundo a distância euclidiana que aproxima ou forma o núcleo de um grupo. 
Aquelas que se distanciam dos padrões médios da sua própria classificação de atividade econômica recebem valores do FAP calculados segundo a sua soma das distâncias padronizadas até o centro do grupo. As empresas cujos perfis demonstrarem morbidade específica menor poderão obter redução das alíquotas de imposto em até $50 \%$ ao conseguir reduzir os encargos previdenciários por morbidade entre seus empregados ${ }^{2}$.

A Resolução citada prevê revisões periódicas que permitirão determinar ajustes de sensibilidade e especificidade dos instrumentos e das técnicas de avaliação empregadas no cálculo do FAP. Pretende-se, com as medidas adotadas, modular o imposto sobre os riscos encontrados nos ambiente de trabalho, para cumprir disposições legais que determinam a redução ou agravo dos mesmos em função do sucesso de medidas de promoção da saúde e de prevenção de doenças e acidentes colocadas em prática pelas empresas.

O objetivo deste artigo é analisar a validade das premissas da determinação do FAP, discutir sua aplicabilidade e potencial para atingir o controle dos riscos decorrentes do modo de produção e dos ambientes de trabalho.

\section{Método}

A montagem do FAP é verificada no presente trabalho com auxílio da literatura epidemiológica clássica. Analisa-se a consistência do método adotado para selecionar as doenças, lesões e acidentes que compõem os grupos de morbidade destinados a construir os vetores de freqüência, gravidade e custo.

Discute-se, à luz do texto e anexos da Resolução 1.236, a validade da aplicação dos conceitos epidemiológicos à situação geradora de morbidade relacionada com o trabalho, bem como suas prováveis conseqüências.

\section{Construção dos Grupos de Morbidade - Listas ou Critérios Epidemiológicos?}

As notificações de doenças e acidentes do trabalho para a Previdência Social no Brasil são objeto de extensa literatura que foge ao escopo dessa apreciação. O próprio texto da Resolução comenta outros autores brasileiros que trabalharam o tema em distintos contextos, apontando para alta subnotificação de doenças e acidentes ${ }^{3}$. Encontra-se, ainda, referência sobre subnotificação dos acidentes do trabalho (AT), estimada em $83,4 \%$ no que se refere aos óbitos ${ }^{4}$. Apontase também $54,1 \%$ de subnotificações com respeito à morbidade e aos acidentes do trabalho do mercado formal ${ }^{5}$.

Os argumentos colocados no texto da Resolução permitem entrever que os órgãos governamentais, por vários motivos, têm dificuldade para cumprir as determinações legais para avaliação e controle das doenças e acidentes nos ambientes de trabalho. Alguns argumentos apontados para isso são: o aspecto social derrogatório para empresas e pessoas que propiciam a ocorrência de acidentes; a recusa em conceder estabilidade após o retorno ao trabalho; a busca da liberdade de despedir o trabalhador em qualquer tempo; a sonegação das contribuições sociais devidas e, fundamentalmente, a recusa em reconhecer a presença dos agentes nocivos causadores de doenças e acidentes.

O último aspecto recai sobre os profissionais especializados no campo da Saúde e Segurança do Trabalho (SST), abrangendo médicos, engenheiros, enfermeiros, psicólogos, e vários outros de nível universitário e técnico-especializado. Cada um deles trabalha usualmente em seus núcleos de competência, mas compartilha o campo multiprofissional com os demais. O reconhecimento da especificidade dos vínculos entre trabalho, doenças e acidentes recai sobre os profissionais de saúde, notadamente os médicos. Os critérios são definidos por consensos científicos, legais e sociais. A demarcação e mutação dos consensos é matéria submetida ao contraditório contínuo e sujeita a visões distintas segundo os referenciais científicos e as visões aplicadas dos conceitos derivados das posições de classe social dos executores ${ }^{6-8}$.

Alternativas distintas têm sido adotadas para a resolução de conflitos sobre a existência de vínculo ou nexo causal (NX) entre doenças, lesões, acidentes e os modos de produção do trabalho. Uma das alternativas é a adoção 
de listas consensuais restritivas, editadas por autoridades governamentais ou associações profissionais de especialistas. Através delas, pretende-se por argumento "de autoridade" recusar outros vínculos estabelecidos pela observação de trabalhadores e profissionais não pertencentes às sociedades de especialidade.

Por meio das listas de morbidade, se, por um lado, um médico do trabalho tem a "liberdade" de fixar um diagnóstico com nexo causal direto ou implícito para um trabalhador doente, por outro lado, agências governamentais, peritos previdenciários, advogados patronais, profissionais de saúde e segurança a serviço patronal, e até seguradoras privadas, podem contestar a existência de nexo causal (NX) em doenças e lesões que não possam ser claramente colocadas na lista. Podem, também, recusar ou discutir a validade da classificação adotada pelo diagnosticador, o que é freqüente.

A característica das listas de doenças é orientar a discussão para a análise individual, caso a caso. Omite-se nessa discussão que as noções de causalidade conhecidas na ciência moderna são fundamentalmente probabilísticas e coletivas ${ }^{9}$. A constituição de listas mínimas de morbidade, que em geral são defendidas para delimitar direitos inalienáveis dos trabalhadores diante dos tribunais, transforma-se em "listas máximas". Não admitem a inclusão de casos individuais que não cumpram os requisitos típicos incluídos e transformam-se em instrumentos de exclusão, ao contrário do propósito com que foram inicialmente construídas e defendidas. Ignoram, em última análise, que todo diagnóstico médico e toda conclusão científica sobre causalidade tem origem em uma conjectura probabilística cuja prova definitiva no nível do indivíduo é filosoficamente impossível.

O contencioso social, previdenciário e jurídico sobre o NX cumpre formalidades de exclusão com recurso freqüente ao determinante que dita a regra de construção do conhecimento científico: Em geral são necessários dados adicionais que fortaleçam a prova de vínculo entre a ocupação e a doença ou lesão. Dados adicionais ou não existem ou são caros e difíceis de serem obtidos. Na dúvida age-se contra e não a favor do réu. O réu nesses casos é, em geral e paradoxalmente, a vítima. O sentimento social de exclusão dos trabalhadores segurados que vêem seus direitos não preservados reflete-se na avaliação negativa da população sobre os benefícios da manutenção dos aparelhos de Estado destinados à saúde e à seguridade social.

Outra estratégia, que não a das listas, é a delimitação de consensos coletivos móveis, com base científica quantitativa ou qualitativa. A delimitação dos problemas relacionando doenças e acidentes com determinadas modalidades de trabalho passa a ser definida em função de argumentos coletivos, conjunturalmente adequados e definidos segundo as regras de probabilidades. Os pesquisadores latino-americanos foram pioneiros no estabelecimento dos consensos entre grupos homogêneos de trabalhadores ${ }^{10-13}$.

O estabelecimento da validade dos critérios consensuais de grupos de trabalhadores expandiu-se para a epidemiologia com o auxílio das constatações de especialistas sobre características que só os trabalhadores e os que freqüentam seus ambientes de trabalho podem reconhecer ${ }^{14}$. Em que pese o desenvolvimento dessa percepção de que o coletivo e o probabilístico deveriam orientar o diagnóstico da existência do NX, as normas oficiais, legais e a atuação dos especialistas costumam negar a habilitação dos não especializados em diagnosticar, dando origem a infindáveis litígios entre profissionais de saúde, previdência, empresas e trabalhadores, que fogem ao escopo da presente análise.

Apresenta-se, portanto, diante desse quadro científico, um avanço social e governamental quando a norma oficial ministerial propõe seguir critérios epidemiológicos quantitativos baseados na unificação de informações pela vinculação de bases de dados oficiais que já existem e não são utilizadas para tal fim. É proposta a união de informações dos sistemas de: Arrecadação e benefícios da Previdência Social; Cadastro Nacional de Informações Sociais - CNIS; Classificação Nacional de Atividades Econômi- 
cas - CNAE-classe; Sistema de Inteligência Fiscal - SIF e Classificação Internacional de Doenças - $10^{a}$ Revisão-CID-10.

Os indicadores de freqüência, gravidade e custo são elaborados a partir do conceito de que a morbidade mais freqüente e que agrava determinado ramo econômico, ou grupo de empresas, é atributo daquela modalidade de trabalho e de produção. Será mais bem sucedido o grupo ou empresa que promover a saúde e reduzir a morbidade encontrada, independentemente da discussão infinita e improdutiva sobre o NX. Será bonificado o sucesso e penalizada a falta de iniciativa, segundo critérios epidemiológicos independentes de listas de morbidade e vínculo ou nexo.

\section{Como medir risco vinculado ao trabalho?}

A proposta da Resolução é incluir como morbidade pertinente todos os diagnósticos iguais, agrupados por categoria da CID-10, cujo Risco Epidemiológico é avaliado pela Razão de Chances, também chamada de 'Odds Ratio' (OR), Razão de Ocorrências, Razão de Produtos Cruzados e várias outras denominações ${ }^{15-16}$.

Encontra-se em PEREIRA, $1995^{15}$, argumento elaborando vantagens e desvantagens da utilização de bases de dados vinculadas, concluindo, em tom de avaliação, que o uso dessa técnica é tradicional nos países escandinavos e na Inglaterra, embora incipiente na América Latina, com relevo para o Brasil.

Para a construção de parâmetros de risco epidemiológico, nesse caso, necessita-se de informações já disponíveis nas bases de dados, a saber: os diagnósticos emitidos pelos médicos que analisam e concedem benefícios da Previdência Social; o ramo e a empresa onde atuam os trabalhadores compensados pelos benefícios; a população trabalhadora empregada, segundo empresa e ramo econômico, em cada ano-calendário. Esses dados são suficientes para construção de incidências e riscos anuais, bem como de chances e suas comparações em razão do tipo $\mathrm{OR}^{17}$.

É interessante notar que o Conselho Nacional de Previdência Social determina o uso de medidas epidemiológicas que equivalem, tanto a incidências quanto a prevalências, pois a população estudada é conhecida para o período de um ano-calendário inteiro.

Ao considerar-se que um trabalhador notificado como presente na relação de 31 de dezembro permanecerá, em média, empregado até 31 de dezembro do ano seguinte, tem-se como provável que a população notificada nesse período representa uma coorte de trabalhadores que experimentará a realização de um ano-pessoa de trabalho, para cada um de seus integrantes. Essa medida permite tratar os benefícios previdenciários como eventos incidentes dentro de cada coorte anual.

Além da coorte, obtém-se a partir das bases de dados oficiais, verdadeiros grupos de casos de doentes e seus respectivos "controles”, que podem ser, nessa circunstância, tanto portadores de outras doenças quanto outros trabalhadores do mesmo ramo econômico produtivo. É possível, segundo essa visão, considerar a análise de coortes, com medidas de OR, ou estudos aninhados de caso-controle, que utilizam as mesmas medidas de $\mathrm{OR}^{16,18,19}$.

Se for feita a opção de análise por coorte, o denominador mais adequado será a população empregada relacionada na CNIS, segundo a classificação econômica da CNAE. Poderá, nesse caso, ser utilizada a medida de OR.

Por outro lado, caso se deseje analisar somente o grupo que demandou e conseguiu benefícios previdenciários por doenças ou acidentes, poder-se-á, alternativamente, usar como comparação ou "controles" o grupo de trabalhadores que iniciou benefícios por diagnósticos diferentes dos analisados em cada cálculo de risco. Nesse caso, a mesma medida de OR deverá ser utilizada.

Emambos os casos, combase populacional empregada ou com controles de outras doenças, os cálculos de risco por medidas de OR devem convergir para valores praticamente idênticos, uma vez que as populações e os grupos de beneficiários ultrapassam a casa de milhão. Os intervalos de confiança para os riscos calculados serão mínimos ou pontuais, 
dadaa pequenavariabilidade denúmeros dessa ordem ${ }^{20}$. Considera-se que o cálculo de OR em população para um ano dispensaria intervalo de confiança por se tratar de um parâmetro para o ano trabalhado. No entanto, ao se extrapolar para freqüências esperadas para o período dos anos seguintes estará sendo feita estimação, tornando-senecessário e pertinente o uso de intervalos de confiança.

Segundo esta visão, poderá ser apontada como doença vinculada à produção ou ao trabalho toda aquela cujo risco epidemiológico, medido por um intervalo de confiança de 99\%, esteja acima do valor unitário. Se o intervalo de confiança não incluir o valor “1,0", essa morbidade ou lesão deverá fazer parte das doenças contabilizadas como objeto de avaliação e controle para a empresa, ou grupo econômico da CNAE.

Sobre as doenças assim selecionadas, será possível avaliar-se o progresso na promoção da saúde e as medidas preventivas contra o adoecimento que deverão ser desenvolvidas para que o FAP seja modificado periodicamente, de acordo com as medidas coletivas e epidemiológicas, nos ambientes de trabalho.

As análises propostas podem ser visualizadas nas Tabelas 1 e 2 . O cálculo dos intervalos de confiança segue as definições propostas por Khan \& Sempos, $1989^{20}$.

Tabela 1 - Análise de risco epidemiológico segundo estratégia de Coorte medindo riscos (OR ou RR) para benefícios por diagnósticos selecionados na população trabalhadora registrada para um ano ou período no calendário. $\left(^{*}\right)$

Table 1 - Epidemiologic risk analysis measuring risks according to a Cohort design (OR or RR) for selected compensated diagnoses from a formally employed population over a year or a calendar period. $(*)$

\begin{tabular}{lccc}
\hline $\begin{array}{l}\text { Atividade } \\
\text { econômica }\end{array}$ & $\begin{array}{c}\text { Diagnóstico com Vínculo } \\
\text { Epidemiológico presumido }\end{array}$ & $\begin{array}{c}\text { População trabalhadora } \\
\text { na atividade estudada }\end{array}$ & Total \\
\hline $\begin{array}{l}\text { Exposta a riscos } \\
\text { (CNAE ou empresa) }\end{array}$ & $\mathrm{A}$ & $\mathrm{B}$ & $\mathrm{A}+\mathrm{B}$ \\
$\begin{array}{l}\text { Outras atividades } \\
\text { não expostas }\end{array}$ & $\mathrm{C}$ & $\mathrm{D}$ & $\mathrm{C}+\mathrm{D}$ \\
Total & $\mathrm{A}+\mathrm{C}$ & $\mathrm{B}+\mathrm{D}$ & $\mathrm{N}$ \\
\hline
\end{tabular}

(*) Método de Katz para intervalo de confiança em Risco Relativo/Katz's Method for Confidence Intervals in Relative Risk. $\mathrm{RR}=\{[\mathrm{a} /(\mathrm{a}+\mathrm{b})] /[\mathrm{c} /(\mathrm{c}+\mathrm{d})]\}$

$\operatorname{Var}(\ln R R)=\{[(b / a) /(a+b)]+[(d / c) /(c+d)]\}$

Tabela 2 - Análise de risco epidemiológico segundo estratégia de Caso-Controle aninhado em Coorte (OR), comparando diagnósticos selecionados com outros diagnósticos conhecidos na população trabalhadora com benefícios iniciados no ano ou no período calendário. ${ }^{*}$ )

Table 2 - Epidemiologic risk assessment (OR) according to a Nested Case-Control design, comparing selected compensated diagnoses with general morbidity among a formally employed workers during a year or a calendar period (*)

\begin{tabular}{lccc}
\hline Atividade & \multicolumn{2}{c}{ Diagnóstico com Vínculo Epidemiológico presumido } & \multirow{2}{*}{ Total } \\
\cline { 2 - 3 } econômica & Sim & Outros diagnósticos & \\
\hline $\begin{array}{l}\text { Exposta a riscos } \\
\text { (CNAE ou empresa) }\end{array}$ & $\mathrm{A}$ & $\mathrm{B}$ & $\mathrm{A}+\mathrm{B}$ \\
$\begin{array}{l}\text { Outras atividades }- \\
\text { não expostas }\end{array}$ & $\mathrm{C}$ & $\mathrm{D}$ & $\mathrm{C}+\mathrm{D}$ \\
Total & $\mathrm{A}+\mathrm{C}$ & $\mathrm{B}+\mathrm{D}$ & $\mathrm{N}$ \\
\hline
\end{tabular}

(*) Pode-se descrever o cálculo das Razões de Chance segundo os seguintes padrões: / ( $\left.{ }^{*}\right)$ Odds Ratio calculation may be described as the following standards:

$\mathrm{OR}=(\mathrm{A} \times \mathrm{D}) /(\mathrm{C} \times \mathrm{B}) ; \mathrm{SE}-\mathrm{In}-\mathrm{OR}=\mathrm{RQ}[(1 / \mathrm{A})+(1 / \mathrm{B})+(1 / \mathrm{C})+(1 / \mathrm{D})]$

Limite inferior do $\ln O R=\ln (\mathrm{OR})-2,57 \times \mathrm{SE}-\ln -\mathrm{OR}$

Limite superior do $\ln O R=\ln (\mathrm{OR})+2,57 \times \mathrm{SE}-\ln -\mathrm{OR}$

$2,57={ }^{\prime \prime} Z$ " ; $99,0 \%$. 


\section{Discussão}

Toda opção metodológica tem vantagens e desvantagens inerentes ao processo. Pereira, $1995^{14}$, conceitua cruzamento de registros e discute "...dificuldades de padronização do dado, à maneira de identificar as pessoas nas diversas fontes e ao perigo potencial de quebra de sigilo dos dados...”. A evasão dos diagnósticos identificados pela codificação dos eventos mórbidos, em trabalhadores com direitos previdenciários, pode ocorrer, no futuro, através da troca das classificações de sítios anatômicos e agentes infecciosos, codificando os eventos atribuíveis às causas externas, segundo seus agentes mecânicos, derivando, para os itens de classificação do grupo XX, os diagnósticos hoje classificados sob outros códigos. A mutação periódica dos critérios médicos de diagnóstico também deverá ser levada em consideração.

Nesse sentido será importante garantir o acompanhamento periódico do desempenho dos códigos de CID-10 adotados para análise e programação do cálculo do FAP.

A subnotifição dos óbitos relacionados com o trabalho deve ser motivo de preocupação ${ }^{3,4}$. Os acidentes não fatais, especialmente aqueles cujo impacto de incapacitação é menor que três dias, fogem às estatísticas acidentárias e aos benefícios previdenciários ${ }^{5}$.

O desempenho de medidas governamentais de avaliação e acompanhamento depende das forças políticas e diretrizes de governo. Antes de 2004, as forças políticas predominantes no Brasil não tinham história de opção pelos critérios epidemiológicos, embora eles estivessem disponíveis há décadas e já existisse competência nacional para sua colocação em prática. O risco que se corre é o desvirtuamento ou a criação de obstáculos políticos e sociais, ainda que travestidos de demandas jurídicas que venham a impedir o comportamento governamental na direção de preservar o interesse coletivo, a saúde e o bem comum. Se isso ocorrer, estaremos diante de lutas em planos políticos que poderão influenciar os profissionais de saúde a negar direitos sociais e adotar visões antagônicas às necessidades de saúde dos trabalhadores, conforme advertência encontradas na literatura $^{6-8}$. Como corolário dessas posições espera-se que as associações de especialistas não se coloquem contrárias à aplicação de critérios probabilísticos, devotando valor total e definitivo às listas de diagnósticos e seus exames complementares individuais de alto custo.

O desenho epidemiológico dos cálculos do FAP não poderá substituir a mobilização coletiva dos trabalhadores e trabalho conjunto e cooperativo dos técnicos dos serviços da seguridade social, do trabalho e da saúde na luta por melhores condições de trabalho. $\mathrm{O}$ objetivo primordial da epidemiologia será o de levar a todos eles informação para decisão; nesse sentido não foi criado ainda melhor instrumento do que a discussão coletiva para ação nos termos da produção epidemiológica desse campo de conhecimento ${ }^{10-13}$. Evidências do campo da Ergonomia dão apoio à afirmação de que a aproximação epidemiológica quantitativa proposta pelo FAP não substituirá elementos subjetivos a serem apreciados dentro das abordagens sociais que são tornadas possíveis pela ergonomia, pela discussão em grupos homogêneos e pela ação coletiva ${ }^{14}$.

Conclui-se que o método proposto para determinação do FAP atende aos requisitos epidemiológicos básicos de definição e validade, assegurada sua revisão periódica para questões de sensibilidade e especificidade. Alerta-se, entretanto, que não será possível confiar-se unicamente nos métodos como instrumentos unilaterais de ação social para avaliação, controle e correção dos males associados ao trabalho, nas modalidades de produção do nosso desenvolvimento social. Os métodos são instrumentos combinados com a decisão e com a ação política que se deseja imprimir, especialmente em um país no qual a agenda política discutida, no ano em que a norma está exarada, é o de promover o desenvolvimento sustentável, através da produção mais limpa, de ambientes de trabalho mais seguros e saudáveis, de liberdade para a organização social e de uma Previdência Social solidária, sustentável, equilibrada e determinada pelas exigências da seguridade social, como direito universal dos cidadãos. 


\section{Referências}

1. Brasil, Ministério da Previdência Social, Conselho Nacional de Previdência Social, Resolução nº 1.236 de 28 de abril de 2004. Diário Oficial da União, Imprensa Nacional, seção I, Brasília, D.F. Segunda-feira, 10 de maio de 2004; edição no 88:36. ISSN: 1677-7042.

2.. Oliveira PRA. O Fator de Ajuste Previdenciário. Seminário Internacional Acidente de Trabalho Trabalho, saúde e qualidade de vida. MPS, Curitiba, Brasil, 9-10 de agosto de 2004.

3. Waldvogel BC. Acidentes do trabalho: os casos fatais, a questão da identificação e da mensuração. Coleção PRODAT Estudos e Análises v.1., n.1., mar-2002, FUNDACENTRO/Segrac, São Paulo, SP, 2002.

4. Hennington EA, Cordeiro R, Moreira Filho, DC. Trabalho, violência e morte em Campinas, São Paulo, Brasil. Cad Saúde Pública 2004; mar-abr, 20(2): 610-7.

5. Binder MCP, Cordeiro R. Sub-registro de acidentes do trabalho em localidade do Estado de São Paulo, 1997. Rev Saúde Pública 2003; 37(4): 409-16.

6. Breilh J, Granda E. Saúde na sociedade. São Paulo: Instituto de Saúde - Secretaria Estadual de Saúde de São Paulo/ABRASCO, 1986. p.40-59, 182-99.

7. Breilh JP. Epidemiologia: economia medicina y política. Santo Domingo, República Dominicana: Ediciones SESPAS, 1980.

8. Breilh J. Nuevos conceptos y técnicas de investigación: guía pedagógica para un taller de metodologia (epidemiologia del trabajo). Serie "Epidemiología Crítica” no 3. Centro de Estudios y Asesoría en Salud CEAS - Quito, Equador; 1994.

9. Rothman K, Greenland S. Causation and causal inference. In: Modern Epidemiology. $2^{\text {nd }}$. Edition, Lippincott Williams \& Wilkins, Philadephia; 1998. p.7-28.

10. Oddone I, Marri G, Gloria S, Briante, G, Chiattella M, Re.. Ambiente de trabalho - a luta dos trabalhadores pela saúde. São Paulo: Editora Hucitec; 1986.

11. Laurell AC. Processo de trabalho e saúde. Saúde em Debate 1981; (11): 8-22.
12. Laurell AC. A saúde-doença como processo social. In: Nunes ED (org.). Textos 3 - Medicina social: aspectos históricos e teóricos. São Paulo: Global Editora; 1983. p.154-5.

13. Laurell AC, Noriega M.. Processo de produção e saúde: trabalho e desgaste operário. São Paulo: Editora Hucitec; 1989..

14. Ferreira LL, Iguti AM. O trabalho dos petroleiros: perigoso, complexo contínuo e coletivo. Scritta Editora (Pensieri); 1996.

15. Pereira MG. Morbidade. In: Epidemiologia: teoria e prática. Rio de Janeiro: Guanabara Koogan S.A; 1995. p.88.

16. Rodrigues L, Werneck GL. Estudos caso-controle. In: Medronho RA, Carvalho DM, Bloch KV, Luiz, RR Werneck GL. Epidemiologia. São Paulo: Atheneu; 2002. p.175-90.

17. Szklö M, Javier Nieto F. Apendix A: A-4_Odds ratio (unmatched and matched). In: Epidemiology: beyond the basics. Gaithersburg, MD: Aspen Publication; 2000. p.440-1.

18. Kleinbaum DG, Kukpper LL, Morgenstern H. Typology of observational study designs. - 5.2. Hybrid designs. In: Epidemiologic research. New York: Van Nostrand Reinhold Co.; 1982. p.70-1.

19. Kelsey JL, Whittemore, AL, Evans AS, Thompson WD. Case-Control studies: II. Further design considerations and analysis. In: Methods in observational epidemiology. Monographs in Epidemiology and Biostatistics, Volume 26. $2^{\text {nd }}$. Edition. New York: Oxford University Press; 1996. p.221-6.

20. Kahn HÁ, Sempos CT. Relative risk and Odds Ratio. In: Statistical methods in epidemiology. Monographs in epidemiology and biostatistics, Volume 12. New York Oxford University Press; 1989. p.56-64.

Recebido em: 03/01/05 Aprovado em: 26/10/05 